\section{Arterite de Takayasu e gravidez}

\section{Takayasu's arteritis and pregnancy}

Abstract Takayasu arteritis is a chronic, idiopathic, inflammatory disease that primarily affects large vessels, such as the aorta and its main branches. This disease is a rare cause of hypertension in women in the menachme. This article discusses multidisciplinary clinical approach to the management of pregnant women with such disease.

Key words Takayasu's arteritis, Vasculitis, Pregnancy
Renata Rodrigues Teixeira de Castro 1

Lino Sieiro Netto 2

Mozart Netto 3

Eduardo Micmacher 4

1-4 Serviço de Clínica Médica do Hospital Geral de Nova Iguaçu.

Av. Henrique Duque Estrada Meyer, 953. Posse. Nova Iguaçu, Rio

de Janeiro, Brasil. CEP: 26.030-380. E-mail: castrorrt@aol.com

Resumo A arterite de Takayasu é uma doença inflamatória crônica, idiopática que afeta a grandes vasos como a aorta e seus principais ramos. Esta doença é uma causa rara de hipertensão em mulheres em idade fértil. Este artigo discute a abordagem clínica multidisciplinar necessária ao acompanhamento de gestantes com esta síndrome.

Palavras-chave Arterite de Takayasu, Vasculite, Gravidez 


\section{Introdução}

A arterite de Takayasu (AT) é uma doença inflamatória sistêmica crônica, idiopática, que afeta grandes vasos como a aorta e seus principais ramos. 1 Tal afecção acomete principalmente mulheres em idade fértil, sendo uma das causas de hipertensão na gestação. Dessa forma, torna-se importante o entendimento de seus métodos diagnósticos, tratamento e complicações. Mesmo nos dias de hoje ainda é freqüiente que a primeira aferição de pressão arterial da mulher seja feita quando a gestante procura o obstetra para acompanhamento pré-natal. Gestantes com hipertensão arterial grave, já no início da gestação serão encaminhadas ao cardiologista, ou ao clínico geral. Tais profissionais deverão estar atentos para a possibilidade de AT, agilizando o processo diagnóstico e de tratamento desses casos, permitindo a ocorrência de gestações mais bem sucedidas.

\section{Incidência}

Estima-se a incidência anual de AT em 2,6 casos por milhão de pessoas, ${ }^{1}$ sendo as mulheres dez vezes mais acometidas do que os homens. ${ }^{2}$ Os primeiros casos descritos ocorreram em países asiáticos, e inicialmente acreditava-se ser uma doença restrita a esses povos.1,3 Entretanto, nas últimas décadas muitos outros casos foram descritos em todo o resto do mundo. 4

\section{Manifestações clínicas}

Alterações em grandes vasos ou presença de hipertensão arterial associados à isquemia de extremidades ou vísceras, mialgias, artralgias, sudorese noturna ou febre, principalmente quando encontrados em pacientes jovens, com aumento da velocidade de hemossedimentação (VHS) devem aumentar o índice de suspeição para o diagnóstico de AT.2 Entretanto, muitos pacientes não apresentam qualquer sintoma constitucional ou vascular, e até $50 \%$ dos pacientes podem apresentar valores normais de VHS. 2

Séries de casos publicadas consideraram a hipertensão arterial um dos achados mais freqüentes nesta síndrome, variando entre $33 \%$ e $60 \% .3,5 \mathrm{~A}$ maioria dos casos de hipertensão descritos foram relacionados ao acometimento (estenose) das artérias renais.3,6 Quando não há estenose dessas artérias, outros mecanismos propostos para a hipertensão são a diminuição da complacência vascular e a disfunção de barorreceptores. 7,8
Independente dos diferentes mecanismos propostos para a ocorrência da hipertensão arterial, o médico deverá estar atento para a presença concomitante de doença oclusiva nas artérias periféricas destas pacientes. Tal fato pode falsear valores elevados de pressão arterial quando aferidos não-invasivamente pelos métodos usuais. A aferição da pressão arterial em membros inferiores tem sido indicada como opção nestas pacientes. Entretanto, raramente, poderá ocorrer acometimento arterial também nos membros inferiores. Nesses casos aconselha-se que a pressão arterial central seja determinada, com o objetivo de avaliar a confiabilidade das medidas nãoinvasivas.1,3,9 Além disso, recomenda-se a monitorização da pressão aórtica central durante o ato anestésico no peri-parto de pacientes cuja pressão arterial não possa ser aferida em qualquer uma das extremidades. ${ }^{3}$

\section{Diagnóstico}

Não há qualquer marcador sorológico capaz de confirmar o diagnóstico de AT. Trabalhos iniciais estudaram a associação de tipos específicos de antígenos de histocompatibilidade com a AT, sem sucesso. 3,10 O VHS, apesar de inespecífico, mostrou-se com grande sensibilidade para tal afecção. ${ }^{1,2}$

Métodos de imagem são os mais utilizados para confirmar o diagnóstico da AT. A angiografia da aorta e seus principais ramos é considerada exame padrão-ouro para tal diagnóstico.1,11-15 Entretanto, ainda não existe qualquer método capaz de diferenciar a doença em seus estados de remissão e atividade. Em gestantes, a realização de angiografia representa um grande risco, em função da exposição à radiação. Nestes casos, a angioressonância representa uma excelente opção diagnóstica.16 Yamada et al. 17 comprovaram a acurácia da angiorressonância no diagnóstico definitivo da AT, descrevendo $100 \%$ de sensibilidade e especificidade para o método.

\section{Morbidade}

Kerr et al. 3 entrevistaram 34 pacientes a respeito dos efeitos da AT, a longo prazo, em sua vida cotidiana. Desses, 26\% negaram qualquer alteração em seu cotidiano. Um segundo grupo de pacientes $(26 \%)$ relatou interrupções no curso usual de suas atividades de acordo com os períodos de atividade da doença. Finalmente, $48 \%$ dos entrevistados apresentava incapacidade permanente para realização de suas atividades cotidianas. 


\section{Tratamento}

O tratamento da atividade da doença é realizado com corticosteróides (prednisona, dose inicial de $1 \mathrm{mg} / \mathrm{kg} / \mathrm{dia}$, seguido de redução gradual). Ocorre remissão após cerca de 22 meses em cerca de $52 \%$ dos pacientes. ${ }^{3}$ Quando não ocorre remissão da doença, ou não é possível a redução da dose de glicocorticóides sem agravamento do quadro, podem ser associados agentes citotóxicos que apresentam sucesso em 1/3 dos casos, porém são contra-indicados na gestação. 3 A normalização do VHS e a melhora clínica são critérios para definição do período de remissão. ${ }^{3}$ Entretanto, apenas $56 \%$ dos pacientes em remissão apresentavam valores normais de VHS no estudo de Kerr et al. 3

$\mathrm{O}$ tratamento cirúrgico pode ser utilizado na correção de estenoses vasculares decorrentes da AT. As principais indicações são: hipertensão secundária à estenose de artéria renal; isquemia de extremidade, limitando atividades cotidianas; manifestações clíni- cas de isquemia cerebrovascular ou estenose maior que $70 \%$ de pelo menos três vasos cerebrais; regurgitação aórtica moderada; isquemia cardíaca associada à estenose coronariana comprovada. ${ }^{3}$

Wang et al. 13 publicaram relato de caso onde foi realizado angioplastia com colocação de stent para correção de estenose de aorta abdominal por AT. Houve controle dramático da hipertensão após a realização do procedimento. 13

\section{Complicações durante a gestação}

Conforme já comentado, a AT acomete principalmente mulheres jovens, em idade fértil. Algumas séries de casos publicadas enfocaram o prognóstico de gestações em pacientes com AT.3,9,11,18-22 Analisando a Tabela 1 pode-se observar que a maioria das gestações em pacientes com AT é bem sucedida, com a ocorrência de nascidos vivos variando entre $70 \%$ e $100 \%$ dos casos.

Tabela 1

Características das gestações relativas à proporção de nascidos vivos em pacientes com arterite de Takayasu: séries de casos.

\begin{tabular}{lccrr}
\hline Publicação & $\begin{array}{c}\text { Número de casos } \\
\text { estudados }\end{array}$ & $\begin{array}{c}\text { Número } \\
\text { de gestações }\end{array}$ & \multicolumn{2}{c}{$\begin{array}{c}\text { Nascidos vivos } \\
\mathbf{n}(\%)\end{array}$} \\
\hline Sharma BK. et al., 2000.11 & 124 & 24 & 17 & $(70,0)$ \\
Bassa A et al., 1995.18 & 3 & 3 & 3 & $(100,0)$ \\
Kerr GS et al., 1994.3 & 60 & 5 & 5 & $(100,0)$ \\
Aso T et al., 1992.19 & 15 & 23 & 19 & $(82,0)$ \\
Matsumura A et al., 1992.20 & 18 & 22 & $22 \quad(100,0)$ \\
Wong VC et al., 1983.21 & 13 & 30 & 26 & $(86,6)$ \\
Ishkawa K, Matsuura S, 1982.22 & 27 & 33 & $33 \quad(100,0)$ \\
Total & 260 & 140 & 125 & $(89,3)$ \\
\hline
\end{tabular}


Matsumura et al. 20 estudaram o comportamento da proteína $\mathrm{C}$ reativa e da pletismografia digital em 22 gestações de pacientes com AT. Nesse estudo concluiu-se que a gestação é um estado favorável à AT, pois houve diminuição da atividade inflamatória e do estado hemodinâmico dessas pacientes. Especulou-se que tal fato poderia estar associado à progesterona, mas isto ainda não foi confirmado. Além desse estudo, relatos de caso isolados 12,18,23-28 descrevem gestações sem intercorrências em pacientes com AT. Chua et al. 27 descreveram um caso de paciente com AT cuja gestação foi conseguida após indução com clomifeno. Além de hipertensão (controlada com propranolol), não houve quaisquer outras complicações durante essa gestação.

Entretanto a exacerbação dos sintomas da AT pode também ser facilitada pela gestação. Sua ocorrência varia entre $25 \%$ e $60 \% .3,24$ Dentre as possíveis complicações descritas neste grupo de gestantes, as principais são: pré-eclampsia, 11,24,29 insuficiência cardíaca, 11 acidente vascular cerebral hemorrágico, ${ }^{22,29}$ crescimento intra-uterino retardado, 11 abortamento espontâneo. ${ }^{21}$ A ocorrência de complicações durante a gestação em pacientes com AT está associada ao envolvimento da aorta abdominal pela doença e ao atraso na procura de acompanhamento médico. ${ }^{11} \mathrm{Na}$ revisão de literatura, apenas um estudo11 indicou realização de abortamento em quatro das 23 gestações acompanhadas, por atividade da doença ainda no primeiro trimestre da gestação.

\section{Controle da hipertensão durante a gestação}

O controle da hipertensão em gestantes com AT é semelhante àquele em pacientes sem tal co-morbidade. Gestantes com hipertensão arterial crônica deverão ser medicadas caso apresentem pressão arterial diastólica igual ou acima de $100 \mathrm{mmHg}$. A metildopa continua sendo a droga de escolha para início do tratamento $($ dose inicial $=250 \mathrm{mg}, 12 / 12 \mathrm{~h}$; dose máxima $=3 \mathrm{~g} /$ dia $)$. O objetivo do tratamento deverá ser a manutenção da pressão arterial diastólica entre 80 e $100 \mathrm{mmHg}$. Diuréticos podem ser mantidos du- rante a gestação. Inibidores da enzima conversora de angiotensina estão contra-indicados neste período. Além de existirem relatos de insuficiência renal fetal e neonatal, tal medicação pode agravar a hipertensão das pacientes com AT uma vez que muitas delas têm estenose de artéria renal ${ }^{3}$ como a principal causa da hipertensão.

Outro fato que deve ser levado em consideração durante o acompanhamento clínico de gestantes hipertensas com AT, diz respeito à aferição da pressão arterial, conforme já mencionado. A aferição em membro sem estenose arterial deverá ser realizada e utilizada para avaliação da resposta ao tratamento. A aferição da pressão arterial em membro com estenose arterial poderá indicar uso de antihipertensivos em altas doses, com queda demasiada da pressão arterial, predispondo à hipoperfusão de órgão como a placenta e o cérebro.

A abordagem do anestesiologista durante o trabalho de parto de gestantes com AT também deverá ser diferenciada, principalmente no que diz respeito à hipertensão e sua monitorização adequada. Clark e al-Qatari ${ }^{30}$ utilizaram a eletroencefalografia durante a cesariana com anestesia geral para monitorizar sinais de isquemia cerebral em uma paciente com AT. Essa paciente havia apresentado acidentes vasculares cerebrais isquêmicos prévios e mantinha severa estenose carotídea bilateral.

\section{Conclusões}

A primeira aferição da pressão arterial em mulheres jovens freqüentemente ocorre durante o acompanhamento pré-natal. A constatação de níveis pressóricos elevados no primeiro trimestre da gestação deve ser seguida por investigação diagnóstica acurada. A arterite de Takayasu deve ser considerada no diagnóstico diferencial destes casos e pode ser confirmada através da angiorressonância (quando houver suspeita clínica compatível). Quando confirmado, sugerimos que o acompanhamento pré-natal seja realizado por diferentes especialistas (obstetra, cardiologista, reumatologista e anestesiologista). 


\section{Referências}

1. Bartholomew JR, Gray BH. General medical care of the patient with rheumatic disease. Large artery occlusive disease. Rheum Dis Clin North Am 1999; 25: 669-86.

2. Mandell BF, Hoffman GS. Rheumatic diseases and the cardiovascular system. In: Braunwald E, Zipes DP, Libby P, editors. Heart disease: a textbook of cardiovascular medicine. Philadeuphia: WB Saunders; 2001. p. 2199-210.

3. Kerr GS, Hallahan CW, Giordano J, Leavitt R, Fauci AS, Menachem R, Hoffman GS. Takayasu arteritis. Ann Intern Med 1994; 120: 919-29.

4. Lande A, Bard R, Rossi P, Passariello R, Castrucci A. Takayasu's arteritis. A world-wide entity. NY State J Med 1976; 76: 1477-82.

5. Nakao K, Ikeda M, Kimata S, Niitani H, Niyahara M Takayasu's arteritis. Clinical report of eight-four case and immunological studies of seven cases. Circulation 1967; 35: 1141-55.

6. Lupi-Herrera E, Sanchez-Torres G, Marchushamer J, Mispireta J, Horwitz S, Vela JE. Takayasu's arteritis. Clinical study of 107 cases. Am Heart J 1977; 93: 94 103

7. Ask-Upmark E. On the pathogenesis of the hypertension in Takayasu's syndrome. Acta Med Scand 1961; 169 467-77.

8. Abe K, Miyazaki S, Kusaka T, Irokawa N, Aoyagi H. Elevated plasma renin activity in aortitis syndrome. Jpn Heart J 1976; 17: 1-11

9. Winn HN, Setaro JF, Mazor M, Reece EA, Black HR, Hobbins JC. Severe Takayasu's arteritis pregnancy: the role of central hemodynamic monitoring. Am J Obstet Gynecol 1988; 159: 1135-6.

10. Ohtawa T. HLA antigens in arterial occlusive diseases in Japan. Jpn J Surg 1976; 6: 1-8.

11. Sharma BK, Jain S, Vasishta K. Outcome of pregnancy in Takaysu arteritis. Int J Cardiol 2000; 75 Supl 1: S159 62.

12. Del Corso L, Moruzzo D, Agelli M, Pentimone F. Takayasu's arteritis on steroid therapy. Seven years follow-up. Panminerva Med 1999; 41: 355-8.

13. Wang YM, Mak GY, Lai KN, Lui SF. Treatment of Takaysu's aortitis with percutaneous transluminal angioplasty and wall stent - a case report. Angiology 1998; 49: 945

14. Sise MJ, Couniham CM, Shackford SR, Rowley WR. The clinical spectrum of Takayasu's arteritis. Surgery 1988; 104: 905-10.

15. Cantú C. Noninavasive cerebrovascular assessment of Takayasu arteritis. Stroke 2000; 31: 2197-202.
16. Coel M, Saito R, Endo PM. Use of magnetic resonance imaging in the diagnosis of Takayasu's arteritis during pregnancy: a case report. Am J Perinatol 1993; 10: 126-

17. Yamada I, Nakagawa T, Himeno Y, Kobayashi Y, Numano F, Shibuya H. Takayasu arteritis: diagnosis with breathhold contrast-enhanced three-dimensional MR angiography. J Magn Reson Imaging 2000; 11: 481-7.

18. Bassa A, Desai DK, Moodley J. Takayasu's disease and pregnancy. Three case studies and review of literature. S Afr Med J 1995; 85: 107-12.

19. Aso T, Abe S, Yaguchi T. Clinical gynecologic features of pregnancy in Takayasu arteritis. Heart Vessels 1992; Suppl 7: 125-32

20. Matsumura A, Moriwaki R, Numano F. Pregnancy in Takayasu arteritis from the view of internal medicine. Heart Vessels 1992; Suppl: 7: 120-4

21. Wong VC, Wang RY, Tse TF. Pregnancy and Takayasu's arteritis. Am J Med 1983; 75: 597-601.

22. Ishigawa K, Matsuura S. Occlusive thromboaortopathy (Takayasu's disease) and pregnancy. Clinical course and management of 33 pregnancies and deliveries. Am J Cardiol 1982; 50: 1293-300

23. Bloechle M, Bollmann R, Chaoui R, Birnbaum M, Bartho S. Pregnancy in Takayasu arteritis. Z Geburtshilfe Neonatol 1995; 199: 116-9.

24. Fignon A, Marret H, Alle C, Jacquet A, Avigdor S, Descamps P, Body G, Lansac J. Association of Takayasu's arteritis, pregnancy and Still's disease. J Gynecol Obstet Biol Reprod (Paris) 1995; 24: 747-50.

25. Del Corso L, De Marco S, Vannini A, Pentimone F. Takayasu's arteritis: low corticosteroid dosage and pregnancy- a case report. Angiology 1993; 44: 827-31.

26. Ben Zineb N, Zine S, Bellasfar M, Mesaad MJ, Sfar R. Association of Takayasu arteritis, Crohn disease and pregnancy. Rev Fr Gynecol Obstet 1992; 87: 591-3.

27. Chua S, Viegas OA, Tan AT, Ratnam SS. Successful outcome of pregnancy in subfertile patient with severe aortoarteritis (Takayasu's disease). Eur J Obstet Gynecol Reprod Biol 1987; 25: 249-53.

28. De Jonge HJ, Knipscheer RJ, Weigel HM. Takayasu's or pulseless disease in pregnancy. Eur J Obstet Gynecol Reprod Biol 1983; 14: 241-9.

29. Cristalli B, Morice P, Heid M, Levardon M. Takayasu' disease and pregnancy. J Obstet Gynecol Biol Reprod (Paris) 1992; 21: 969-70

30. Clark AG, al-Qatari M. Anaesthesia for cesarean section in Takayasu’s disease. Can J Anaesth 1998; 45: 377-9.

Recebido em 10 de março de 2002

Versão final reapresentada em 20 de junho de 2002

Aprovado em 15 de agosto de 2002 